istry of the cord factor of Mycobacterium tuberculosis. J. Biol. Chem. 214:251-265.

5. Noll, H., Asselineau, J., and Lederer, E. 1956. The chemical structure of the cord factor of Mycobacterium tuberculosis. Biochim. Biophys. Acta. 20:299-309.

6. Goren, M.B. 1972. Mycobacterial lipids: selected topics. Bacteriol. Rev. 36:33-64.

7. Asselineau, J., and Lederer, E. 1950. Structure of the mycolic acids of mycobacteria. Nature. 166:782-783.

8. George, K.M., Yuan, Y., Sherman, D.R., and Barry, C.E., 3rd. 1995. The biosynthesis of cyclopropanated mycolic acids in Mycobacterium tuberculosis. Identification and functional analysis of CMAS- 2 . J. Biol. Chem. 270:2792-2798.

9. Yuan, Y., Lee, R.E., Besra, G.S., Belisle, J.T., and Barry, C.E., 3rd. 1995. Identification of a gene involved in the biosynthesis of cyclopropanated mycolic acids in Mycobacterium tuberculosis. Proc. Natl. Acad. Sci. U. S. A. 92:6630-6634.

10. Barry, C.E., 3rd, et al. 1998. Mycolic acids: structure, biosynthesis, and physiological function. Prog. Lipid Res. 37:143-179.

11. Brennan, P.J., and Nikaido, H. 1995. The envelope of mycobacteria. Annu. Rev. Biochem. 64:29-63.

12. Yuan, Y., YaQi, Z., Crane, D.D., and Barry, C.E., 3rd. 1998. The effect of oxygenated mycolic acid composition on cell wall function and macrophage growth in Mycobacterium tuberculosis. Mol. Microbiol. 29:1449-1458.

13. Dubnau, E., et al. 2000. Oxygenated mycolic acids are necessary for virulence of Mycobacterium tubercu- losis in mice. Mol. Microbiol. 36:630-637.

14. Glickman, M.S., Cox, J.S., and Jacobs, W.R., Jr. 2000 A novel mycolic acid cyclopropane synthetase is required for cording, persistence, and virulence of Mycobacterium tuberculosis. Mol. Cell. 5:717-727.

15. Rao, V., Fujiwara, N., Porcelli, S.A., and Glickman, M.S. 2005. Mycobacterium tuberculosis controls host innate immune activation through cyclopropane modification of a glycolipid effector molecule. J. Exp. Med. 201:535-543.

16. Chatterjee D., and Khoo, K.H. 1998. Mycobacterial lipoarabinomannan: an extraordinary lipoheteroglycan with profound physiological effects. Glycobiology. 8:113-120.

17. Chatterjee D., Roberts, A.D., Lowell, K., Brennan, P. and Orme, I.M. 1992. Structural basis of capacity of lipoarabinomannan to induce secretion of tumor necrosis factor. Infect. Immun. 60:1249-1253.

18. Quesniaux, V.J., et al. 2004. Toll-like receptor 2 (TLR2)-dependent-positive and TLR2-independent-negative regulation of proinflammatory cytokines by mycobacterial lipomannans. J. Immu nol. 172:4425-4434.

19. Brightbill, H.D., et al. 1999. Host defense mechanisms triggered by microbial lipoproteins through toll-like receptors. Science. 285:732-736.

20. Goren, M.B., Broki, O., and Das, B.C. 1976. Sulfatides of Mycobacterium tuberculosis: the structure of the principal sufatide (SL-1). Biochemistry. 15:2728-2735.

21. Camacho, L.R., et al. 2001. Analysis of the phthiocerol dimycocerosate locus of Mycobacterium tuberculo- sis. Evidence that this lipid is involved in the cell wall permeability barrier. J. Biol. Chem. 276:19845-19854.

22. Cox, J.S., Chen, B., McNeil, M., and Jacobs, W.R., Jr. 1999. Complex lipid determines tissue-specific replication of Mycobacterium tuberculosis in mice. Nature. 402:79-83.

23. Rousseau, C., et al. 2003. Virulence attenuation of two Mas-like polyketide synthase mutants of Mycobacterium tuberculosis. Microbiology. 149:1837-1847.

24. Valway, S.E., et al. 1998. An outbreak involving extensive transmission of a virulent strain of Mycobacterium tuberculosis. N. Engl. J. Med. 338:633-639.

25. Manca, C., et al. 1999. Mycobacterium tuberculosis CDC1551 induces a more vigorous host response in vivo and in vitro, but is not more virulent than other clinical isolates. J. Immunol. 162:6740-6746.

26. Sreevatsan, S., et al. 1997. Restricted structural gene polymorphism in the Mycobacterium tuberculosis complex indicates evolutionarily recent global dissemination. Proc. Natl. Acad. Sci. U. S. A. 94:9869-9874

27. Reed, M.B., et al. 2004. A glycolipid of hypervirulent tuberculosis strains that inhibits the innate immune response. Nature. 431:84-87.

28. Shimono, N., et al. 2003. Hypervirulent mutant of Mycobacterium tuberculosis resulting from disruption of the mce1 operon. Proc. Natl. Acad. Sci. U. S. A. 100:15918-15923.

29. Karakousis, P.C., Bishai, W.R., and Dorman, S.E. 2004. Mycobacterium tuberculosis cell envelope lipids and the host immune response. Cell. Microbiol. 6:105-116.

\title{
Probing the role of stearoyl-CoA desaturase-1 in hepatic insulin resistance
}

\author{
Matthew T. Flowers, ${ }^{1}$ Makoto Miyazaki, ${ }^{2}$ Xueqing Liu, ${ }^{2}$ and James M. Ntambi1,2 \\ 1Department of Nutritional Sciences and 2Department of Biochemistry, University of Wisconsin — Madison, Madison, Wisconsin, USA.
}

Previous studies using stearoyl-CoA desaturase-1-deficient (SCD1-deficient) mice have shown that this enzyme plays an important role in many diseases of altered cellular metabolism including obesity, insulin resistance, and dyslipidemia. Although SCD1 activity is highest in lipogenic tissues such as the liver and adipose tissue, it is also present at lower levels in most tissues. To better understand the role of SCD1 in liver metabolism it is necessary to explore SCD1 deficiency in a more focused, tissue-specific manner. This commentary focuses on 2 recent studies published in the JCI that address this question using antisense oligonucleotide inhibition of SCD1. First, Jiang et al. have previously reported that long-term inhibition of SCD1 prevents the development of high-fat diet-induced obesity and hepatic steatosis. Second, Gutiérrez-Juárez et al. show in this issue that short-term inhibition of hepatic SCD1 is sufficient to prevent diet-induced hepatic insulin resistance, signifying an important role of hepatic SCD1 in liver insulin sensitivity (see related article beginning on page 1686).

Nonstandard abbreviations used: ACC, acetyl-CoA carboxylase; AMPK, AMP kinase; ASO, antisense oligonucleotide; MUFA, monounsaturated fatty acid; PTP1B, protein tyrosine phosphatase 1B; SCD, stearoylCoA desaturase; TG, triglyceride.

Conflict of interest: The authors have declared that no conflict of interest exists.

Citation for this article: J. Clin. Invest. 116:1478-1481 (2006). doi:10.1172/JCI28774.
Stearoyl-CoA desaturase (SCD) is the central lipogenic enzyme catalyzing in vivo reactions in the synthesis of monounsaturated fatty acids (MUFAs), particularly oleate (C18:1n-9) and palmitoleate (C16:1n-7), which are the major MUFAs of membrane phospholipids, triglycerides (TGs), wax esters, and cholesteryl esters. Recent studies of SCD1 have yielded many new insights into the biology of lipid metabolism and have demonstrated that mice with a global deletion of $S c d 1$ (Scd1 1/- mice) are resistant to high-fat dietinduced obesity and glucose intolerance (1). Scd1 $1^{-/-}$mice also have increased hepatic fatty acid oxidation and decreased lipogenic gene expression (1). Furthermore, Scd1 $1 /-$ mice exhibit increased thermogenesis and insulin signaling in skeletal muscle and brown adipose tissue (2-4). Currently the mechanisms leading to the phenotypes due to SCD deficiency or overexpression in different tissues are not understood; yet the findings so far reveal that SCD1 is an important metabolic control point in lipid metabolism and is a promising drug target for the treatment of the metabolic syndrome.

\section{Can obesity and symptoms of the metabolic syndrome be alleviated by inhibition of SCD1?}

In vivo antisense oligonucleotide (ASO) reduction of target genes is a powerful tool for 


\section{A Short-term (5 days) SCD1 ASO treatment}

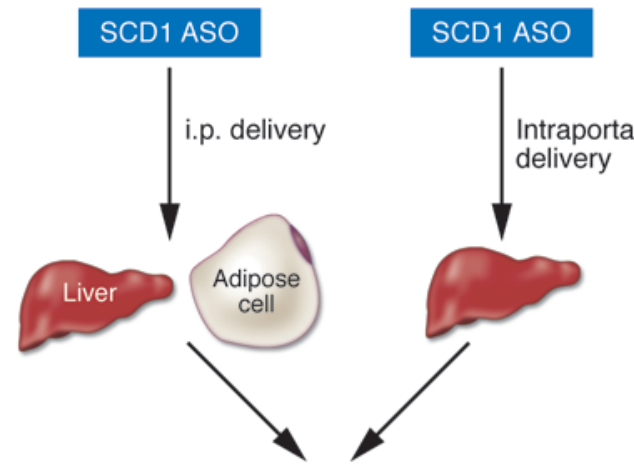

Decreased SCD1 expression

Increased hepatic insulin sensitivity

Decreased diet-induced insulin resistance

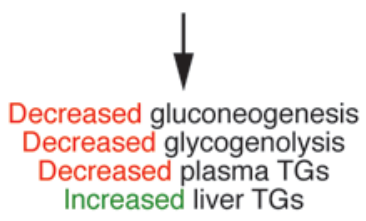

B Long-term (4-10 weeks) SCD1 ASO treatment

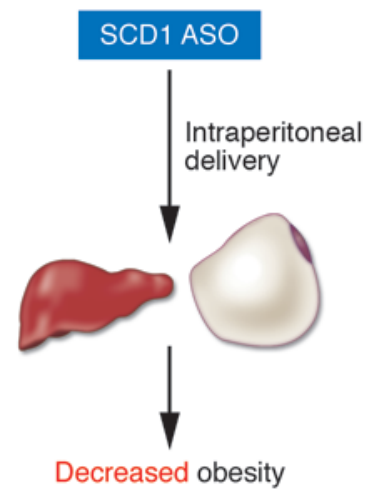

Decreased liver steatosis

Decreased postprandial glucose/insulin

Increased $\mathrm{O}_{2}$ consumption/activity

\section{Figure 1}

Metabolic effects elicited by inhibition of SCD1 by ASOs. The inhibition of SCD1 in rodents with ASOs has been shown to prevent many high-fat diet-induced metabolic complications. i.p. delivery of SCD1 ASO results in decreased SCD1 expression in liver and adipose in both short-term (A) and long-term treatment periods (B). In studies by Gutiérrez-Juárez et al., shortterm treatment (5 days) with i.p. SCD1 ASO prevented diet-induced insulin resistance (10) (A). In these studies, short-term liver-specific intraportal SCD1 ASO treatment also elicited these effects (A). Long-term treatment (4-10 weeks) with i.p. SCD1 ASO prevents diet-induced obesity and hepatic steatosis (9) (B). Adipose-specific inhibition as well as long-term liver-specific inhibition of SCD1 remain to be explored (A and $\mathbf{B})$.

identifying novel metabolic drug targets and further elucidating the role of various genes in cellular metabolic pathways. The use of ASO-mediated inhibition in adult mice also allows for manipulation of gene expression of targets that are necessary during embryonic development. This is exemplified by recent studies investigating ASO-mediated inhi-

\section{SCD1 ASO}

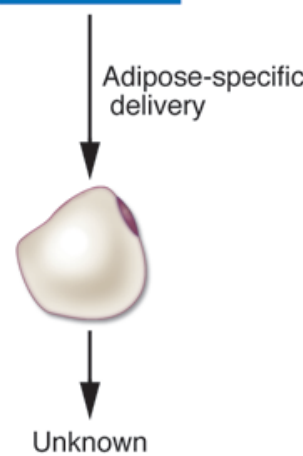

SCD1 ASO

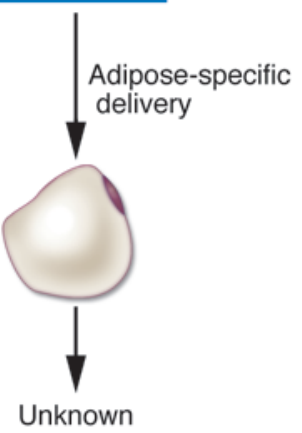

further mechanistic insight into results previously observed in whole-body $S c d 1^{-/-}$gene knockout studies. Similar to the results obtained in Scd1 $1^{-/-}$mice, Jiang et al. reported that ASO-mediated inhibition of SCD1 in $\mathrm{C} 57 \mathrm{BL} / 6 \mathrm{~J}$ mice resulted in resistance to high-fat diet-induced obesity and hepatic steatosis (9). These animals also had lower postprandial glucose and insulin levels, increased oxygen consumption, increased physical activity, and reduced lipogenic gene expression (Figure 1). A benefit of this ASO-mediated approach is that it avoids the deleterious effects of SCD1 deficiency in tissues such as the skin, which results in alopecia and skin abnormalities. Although more localized than global deletion of a gene, i.p. delivery of ASO inhibitors does not allow for the unequivocal determination of the tissuespecific role of target genes because i.p. injection of Scd1-targeted ASOs caused decreased Scd1 gene expression in brown and white adipose tissue as well as in liver $(9,10)$ and possibly in other unidentified tissues.

Unlike the previous studies by Jiang et al. (9), which involved a 10-week ASO-mediated treatment and high-fat (lard) diet feeding, the studies reported by Gutiérrez-Juárez et al. in this issue of the JCI (10) focused on the early stages of hepatic insulin resistance elicited by a shorter-term feeding of a high-fat diet. In these studies the metabolic consequences of ASO-mediated inhibition of SCD1 were investigated prior to changes in body weight, which occur with longer feeding periods. Whereas control mice and rats have increased hepatic glucose production and decreased insulin-mediated inhibition of hepatic glucose production when fed the high-fat diet, animals treated with the $S c d 1$-targeted ASO are resistant to these diet-induced effects (Figure 1). Therefore, these studies clearly illustrate that hepatic SCD1 activity is involved in the development of diet-induced hepatic insulin resistance in these animals.

\section{Do dietary and de novo MUFAs interact to regulate metabolism?}

SCD1 deficiency poses an interesting physiological question. The primary lipogenic tissues such as liver and white adipose tissue synthesize MUFAs via SCD1. These tissues subsequently release MUFAs into the circulation via lipoprotein cholesteryl esters, TGs, and phospholipids and also as free fatty acids. In $S c d 1^{-/-}$mice and in i.p. $S c d 1$ targeted ASO-treated mice and rats, the contribution of liver and adipose tissue to MUFA production is severely inhibited and there is decreased exposure of other periph- 
eral tissues to circulating MUFAs derived from de novo synthesis. In the situation of liver-specific SCD1 inhibition as achieved by intraportal ASO delivery in the studies of Gutiérrez-Juárez et al. (10), adipocytederived MUFAs would potentially influence the fatty composition of the liver. Despite the availability of MUFAs from adipose tissues as well as from the oleate-rich lard diet, liver-specific SCD1 inhibition elicited effects on hepatic insulin signaling (10). Since previous studies of the role of SCD1 on insulin signaling were mostly based on the chronic effects observed in $S c d 1^{-/-}$mice, the observations from Gutiérrez-Juárez et al. provide a direct link between SCD1 deficiency and the improvement of insulin action in liver (10).

Surprisingly, the hepatic TG phenotype observed differs among the studies of Gutiérrez-Juárez et al. (10), those of Jiang et al. (9), and studies using $S c d 1^{-1-}$ mice $(1,11)$. Whereas a 10-week treatment of mice with $S c d 1$ targeted ASO and high-fat diet resulted in reduced hepatic steatosis, the current study shows that short-term treatment with Scd1targeted ASO results in increased hepatic TG levels relative to both high-fat-and standard chow-fed animals treated with the control ASO. This suggests that improved insulin signaling due to SCD1 deficiency may occur in the absence of decreased cellular TG levels. A potential mechanism for the increased hepatic TG levels is the acute enhancement of insulin signaling. The overfed SCD1 ASO group displayed increased Akt phosphorylation and dramatic suppression of glucose6-phosphatase and phosphoenolpyruvate carboxykinase gene expression relative to both the standard chow and overfed control ASO groups. This alteration in insulin signaling could affect both the synthesis and the secretion of hepatic TGs. Decreased hepatic glucose cycling due to transcriptional repression of gluconeogenic genes and the stimulatory effect of insulin signaling on glycolysis may augment the conversion of glucose and gluconeogenic precursors into acetyl-CoA for de novo fatty acid synthesis. However, the levels of the lipogenic enzymes ACC and fatty acid synthase were not increased and tended to be lowered by Scd1-targeted ASO treatment relative to the other groups (10). Additionally, decreased hepatic SCD1 expression in the SCD1 ASO group should result in decreased production of MUFAs, which are the preferred substrate for TG production (11). It is possible that the partial repression of SCD1 activity by ASO-mediated inhibition is permissive for hepatic TG synthesis due to adequate MUFA production, unlike the $S c d 1^{-/-}$mouse, which has a more severe reduction in MUFA synthesis.

The accumulation of hepatic TGs may also be due to increased insulin-mediated inhibition of VLDL-TG secretion. Hepatic insulin signaling decreases VLDL-TG secretion, and dysregulation of this mechanism contributes to the dyslipidemia observed in insulin-resistant states (12). Thus increased insulin signaling elicited by hepatic SCD1 deficiency may decrease both hepatic glucose and VLDL-TG output, resulting in hepatic accumulation of TGs. Additionally, there may be species differences between rats and mice that influence how SCD1 deficiency influences metabolism. Although the mechanism by which SCD1 deficiency improves insulin signaling is not known, Gutiérrez-Juárez et al. found that Scd1-targeted ASO treatment decreased hepatic protein tyrosine phosphatase $1 \mathrm{~B}$ (PTP1B) protein levels, which was similar to the decrease observed in the muscle of $\mathrm{Scd1} 1^{-/}$ mice $(2,10)$. These data suggest that SCD1 and/or its product MUFAs may directly regulate PTP1B expression. Future experiments will be required to address this hypothesis. In contrast to the increased level of AMP kinase (AMPK) phosphorylation observed in the livers of $S c d 1^{-/-}$mice (13), short-term treatment with Scd1-targeted ASOs did not affect AMPK phosphorylation (10), suggesting that increased insulin sensitivity by SCD 1 deficiency may be independent of AMPK activation.

Although Gutiérrez-Juárez et al. show that treatment with Scd1-targeted ASOs restores the ability of insulin to suppress hepatic glucose production in overfed animals, this study did not determine whether metabolic differences are also observed in chow-fed animals (10). $S c d 1^{-/}$mice are not only resistant to many high-fat diet-induced phenomena that develop in wild-type animals with normal SCD1 activity, but also display numerous metabolic changes in liver, adipose tissue, and muscle when maintained on a chow diet $(1-4,11,13)$. Therefore, future studies will be necessary to address a key unsettled issue of the role of SCD1 in hepatic insulin signaling. Is hepatic SCD1 deficiency elicited by ASO-mediated inhibition of SCD1 only preventative for the onset of diet-induced hepatic insulin resistance, or does hepatic SCD1 deficiency also augment insulin signaling relative to control animals in the absence of a dietary stress?

\section{SCD1 inhibition: where, how much, and for how long?}

Many questions remain in the elucidation of the tissue-specific role of SCD1. First, where is SCD1 deficiency most important for eliciting metabolic effects? The current studies by Gutiérrez-Juárez et al. suggest that hepatic SCD1 deficiency alone is sufficient to enhance hepatic insulin sensitivity (10). However, the inability of the i.p. ASOmediated inhibition of SCD1 to enhance whole-body glucose uptake suggests that SCD1 expression in tissues other than liver and adipose also contribute to enhancing the action of insulin. SCD1 expression in muscle may be most important for this phenotype, since this tissue is the major site of glucose disposal. In support of this hypothesis, increased expression of SCD1 in skeletal muscle causes abnormal lipid metabolism and may contribute to the onset of obesity and insulin resistance in humans (14). The correlation of SCD 1 activity with metabolic phenotypes highlights another key question. How much SCD1 deficiency is necessary to elicit local or whole-body metabolic changes? Although $\mathrm{Scd} 1^{-/-}$mice have pronounced phenotypes relative to $S c d 1^{+/+}$animals, current SCD1 ASO studies suggest that partial SCD1 deficiency also alters metabolism.

More research is also needed to determine the tissue-specific contribution of SCD1 in controlling other phenotypes observed in $S c d 1^{-/-}$mice, such as resistance to dietinduced obesity, increased metabolic rate, and increased food intake. Additionally, how long is SCD1 deficiency required to elicit metabolic effects? Would liver-selective SCD1 inhibition by intraportal ASO delivery elicit resistance to obesity in longterm diet studies, or are extrahepatic tissues more important for this phenotype? If not, would hepatic insulin sensitivity persist in an obese animal with liver-selective deficiency of SCD1? Although the current studies did not detect a difference in wholebody glucose disposal, they were performed under conditions of controlled hyperinsulinemia. The role of SCD1 expression in various tissues, including the pancreatic $\beta$ cell, in regulating insulin secretion is not understood. Thus a glucose tolerance test or similar assessment that demands endogenous insulin secretion would be necessary to determine the overall physiological contribution of SCD1 deficiency to wholebody glucose disposal.

In summary, the complications of the metabolic syndrome are intimately related to the dysregulation of lipid metabolism in various tissues. Among the many tissues involved, the liver and adipose are responsible for integrating many complimentary and sometimes conflicting meta- 
bolic stimuli and responding accordingly. The recent studies described herein support an important role for SCD1 in the metabolic response of these tissues and the development of obesity and insulin resistance. The mechanism for how SCD1 or its product, MUFAs, modulate metabolism is unknown. However, the studies by Gutiérrez-Juárez et al. highlight that increased hepatic insulin sensitivity due to liver-specific inhibition of SCD1 may exist independent of body weight and paradoxically in the presence of increased liver TG and long-chain fatty acyl-CoAs (10).

Address correspondence to: James M. Ntambi, University of Wisconsin - Madison, Department of Biochemistry, 433 Babcock Drive, Madison, Wisconsin 53706, USA. Phone: (608) 265-3700; Fax: (608) 2653272; E-mail: ntambi@biochem.wisc.edu.
1. Ntambi, J.M., et al. 2002. Loss of stearoyl-CoA desaturase-1 function protects mice against adiposity. Proc. Natl. Acad. Sci. U. S. A. 99:11482-11486.

2. Rahman, S.M., et al. 2003. Stearoyl-CoA desaturase 1 deficiency elevates insulin-signaling components and down-regulates protein-tyrosine phosphatase $1 \mathrm{~B}$ in muscle. Proc. Natl. Acad. Sci. U. S. A. 100:11110-11115.

3. Lee, S.H., et al. 2004. Lack of stearoyl-CoA desaturase 1 upregulates basal thermogenesis but causes hypothermia in a cold environment. J. Lipid Res. 45:1674-1682.

4. Rahman, S.M., et al. 2005. Stearoyl-CoA desaturase 1 deficiency increases insulin signaling and glycogen accumulation in brown adipose tissue. Am. J. Physiol. Endocrinol. Metab. 288:381-387.

5. Savage, D.B., et al. 2006. Reversal of diet-induced hepatic steatosis and hepatic insulin resistance by hepatic antisense oligonucleotide inhibitors of acetylCoA carboxylase 1 and 2. J. Clin. Invest. 116:817-824. doi:10.1172/JCI27300.

6. Yu, X.X., et al. 2005. Antisense oligonucleotide reduction of DGAT2 expression improves hepatic steatosis and hyperlipidemia in obese mice. Hepatology. 42:362-371.

7. Abu-Elheiga, L., et al. 2005. Mutant mice lacking acetyl-CoA carboxylase 1 are embryonic lethal. Proc. Natl. Acad. Sci. U. S. A. 102:12011-12016.
8. Stone, S.J., et al. 2004. Lipopenia and skin barrier abnormalities in DGAT2-deficient mice. J. Biol. Chem. 279:11767-11776.

9. Jiang, G., et al. 2005. Prevention of obesity in mice by antisense oligonucleotide inhibitors of stearoylCoA desaturase-1. J. Clin. Invest. 115:1030-1038. doi:10.1172/JCI200523962.

10. Gutiérrez-Juárez, R., et al. 2006. Critical role of stearoyl-CoA desaturase-1 (SCD1) in the onset of diet-induced hepatic insulin resistance. J. Clin. Invest. 116:1686-1695. doi:10.1172/JCI26991.

11. Miyazaki, M., Kim, Y.C., Gray-Keller, M.P., Attie, A.D., and Ntambi, J.M. 2000. The biosynthesis of hepatic cholesterol esters and triglycerides is impaired in mice with a disruption of the gene for stearoyl-CoA desaturase 1. J. Biol. Chem. 275:30132-30138.

12. Avramoglu, R.K., Basciano, H., and Adeli, K. 2006. Lipid and lipoprotein dysregulation in insulin resistant states. Clin. Chim. Acta. In press.

13. Dobrzyn, P., et al. 2004. Stearoyl-CoA desaturase 1 deficiency increases fatty acid oxidation by activating AMP-activated protein kinase in liver. Proc. Natl. Acad. Sci. U. S. A. 101:6409-6414.

14. Hulver, M.W., et al. 2005. Elevated stearoyl-CoA desaturase-1 expression in skeletal muscle contributes to abnormal fatty acid partitioning in obese humans. Cell Metab. 2:251-261.

\title{
Cryptococcal virulence: beyond the usual suspects
}

\author{
Aaron P. Mitchell
}

Department of Microbiology, Columbia University, New York, New York, USA.

\begin{abstract}
In this issue of the JCI, the observation of the altered pathogenicity of a Cryptococcus neoformans glucosylceramide (GlcCer) mutant shines new light on the initiation of cryptococcal infection. Rittershaus and colleagues demonstrate that the cell surface glycosphingolipid GlcCer is essential for the fungus to grow in the extracellular environments of the host bloodstream and alveolar spaces of the lung, which, in contrast to the acidic intracellular environment of macrophages, are characterized by a neutral $\mathrm{pH}$ (see the related article beginning on page 1651). Their findings establish an unexpected connection between this glycosphingolipid and the fungal responses to physiological $\mathrm{CO}_{2}$ and $\mathrm{pH}$. They also focus new attention on the therapeutic potential of anti-GlcCer antibodies found in convalescent sera.
\end{abstract}

Cryptococcus neoformans is among the few fungal pathogens with well-defined virulence factors, including a polysaccharide capsule and a melanin coat (1). The recent past has witnessed the identification of many new genes that impact C. neoformans virulence, and in most cases the new genes ultimately govern those known virulence factors. A report from Rittershaus, Del Poeta, and

Nonstandard abbreviations used: Gcs; glucosylceramide synthase; GlcCer, glucosylceramide.

Conflict of interest: The author has declared that no conflict of interest exists.

Citation for this article: J. Clin. Invest. 116:1481-1483 (2006). doi:10.1172/JCI28842. colleagues in this issue of the JCI (2) is thus surprising in that it establishes that a new virulence regulator does not act through any previously known virulence traits, but through a connection between lipid-mediated signaling and the pathogen's response to the $\mathrm{CO}_{2}$ levels and $\mathrm{pH}$ of host tissue. The report provides intriguing new insight into the natural infection process and points to the potential therapeutic significance of an antifungal antibody response.

The connection between GIcCer and C. neoformans virulence

C. neoformans is an opportunistic pathogen that causes disseminated infection and meningoencephalitis in immunocompromised hosts, especially those with AIDS (1). Its close relative, Cryptococcus gattii, is a primary pathogen that caused an outbreak recently on Vancouver Island $(3,4)$. Infection begins with inhalation of airborne spores or yeast cells. The organism is eventually phagocytosed by macrophages, in which it survives as an intracellular pathogen (5). Rittershaus and colleagues show that the poorly understood events that occur between inhalation and macrophage phagocytosis depend upon cryptococcal synthesis of the sphingolipid glucosylceramide (GlcCer) (2).

GlcCer is found at the surface of C. neoformans cells and accumulates at the neck between the mother cell and the emerging daughter cell. In order to determine the function of GlcCer, the authors created a mutant C. neoformans strain lacking GlcCer synthase 1 (Gcs1), which they rigorously show to be encoded by the gcs 1 gene (2). This $\Delta g c s 1$ mutant had an unusual phenotype: it was completely avirulent in mice following nasal inhalation, yet caused lethal infection when delivered through intravenous injection (Figure 1). The inhaled organisms 